\title{
Calibration of double stripe 3D laser scanner systems using planarity and orthogonality constraints
}

\author{
Ş. Ozan, Ş. Gümüştekin* \\ Izmir Institute of Technology, Department of Electrical and Electronics Engineering, Urla, Izmir, 35430, Turkey
}

\section{A R T I C L E I N F O}

\section{Article history:}

Available online 11 September 2013

\section{Keywords:}

3D laser scanner

Scanner calibration

Multi stripe

Plane detection

Nelder-Mead algorithm

\begin{abstract}
A B S T R A C T
In this study, 3D scanning systems that utilize a pair of laser stripes are studied. Three types of scanning systems are implemented to scan environments, rough surfaces of near planar objects and small 3D objects. These scanners make use of double laser stripes to minimize the undesired effect of occlusions. Calibration of these scanning systems is crucially important for the alignment of 3D points which are reconstructed from different stripes. In this paper, the main focus is on the calibration problem, following a treatment on the pre-processing of stripe projections using dynamic programming and localization of 2D image points with sub-pixel accuracy. The 3D points corresponding to laser stripes are used in an optimization procedure that imposes geometrical constraints such as coplanarities and orthogonalities. It is shown that, calibration procedure proposed here, significantly improves the alignment of 3D points scanned using two laser stripes.
\end{abstract}

(C) 2013 Elsevier Inc. All rights reserved.

\section{Introduction}

Reconstruction of 3D surface geometry is an active research area in both computer vision and computer graphics. Due to physical and geometrical varieties of object surfaces, many different application specific scanning systems, methods and algorithms have been developed in order to gather 3D surface geometry.

Stereo imaging methods, especially two-frame stereo methods are widely studied in computer vision. Solving the stereo correspondence problem is the fundamental task in these methods. In [1] a detailed taxonomy and performance analysis of these methods can be found. In traditional stereo imaging, the sensor position is changed where the scene illumination is preferably kept constant. On the contrary, in photometric stereo techniques [2,3], the sensor position is fixed and the scene illuminant position is changed accordingly.

Methods for determining depth from focus [4] require multiple images of the scene taken with different focal parameters, where the depth from defocus methods ([5] and [6]) requires fewer images. In both types of methods the scene is considered as lambertian. For semiglossy dielectric materials, shape from polarization

\footnotetext{
* Corresponding author.

E-mail addresses: sukruozan@iyte.edu.tr (Ş. Ozan), sevketgumustekin@iyte.edu.tr (Ş. Gümüştekin).
}

can be used. In [7] and [8] polarization imaging methods are used to find local surface normals and hence the surface shape.

Another common method to capture 3D information is to use structured light. Using projection of a coded light pattern, it is possible to capture a depth image from a single image frame. Several different coding strategies have been used for generating light patterns [9-11]. Structured light patterns are also shown to be effective when used in conjunction with stereo techniques [12].

The projection of complex light patterns generally requires bulky and expensive projection devices that consume excessive amount of power. In many applications where scanners need to be mounted on a moving platform, simple laser emitters that project light stripes can be used instead. In such implementations, the main problem is the calibration of the light source [13-15].

Laser stripe based scanner systems suffer from a natural side effect if the light source is at a fixed position with respect to camera. A shadow is produced when the light stripe is blocked from the viewpoint by a surface. In this study two independent line lasers are preferred in order to minimize the effect of scene occlusions. This setup is preferable to a single line laser, multiple cameras scenario due to significantly reduced cost and flexibility in different types of scanning problems.

The only constraint imposed here about positioning the two light sources is that the stripes should be visible in different halves of the image frame. This study mainly focuses on the calibration of two independent line lasers with respect to camera reference 


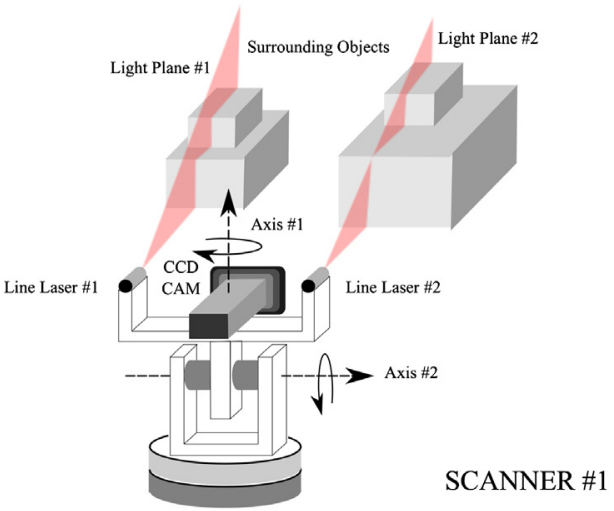

(a)

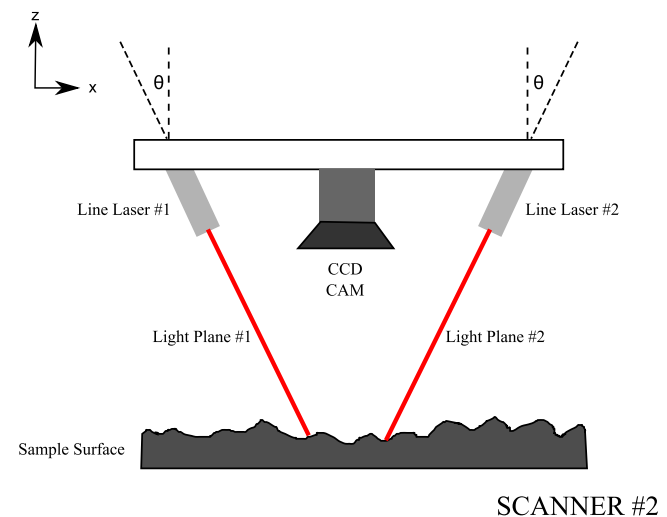

(b)

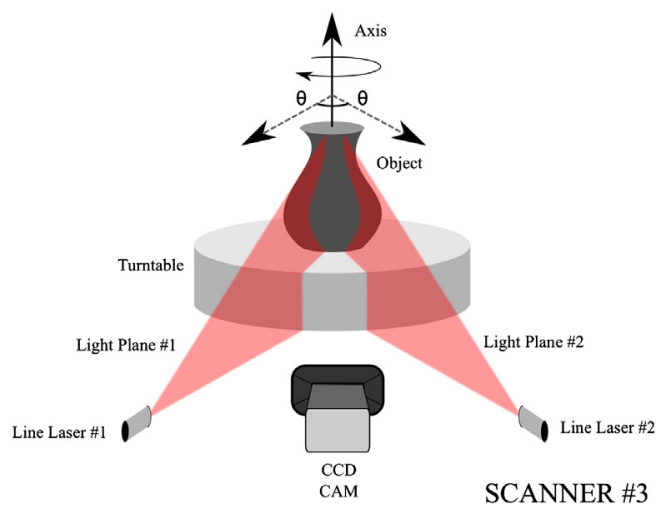

(c)

Fig. 1. (a) Environment scanner (Scanner-1), (b) surface scanner (Scanner-2), (c) object scanner (Scanner-3).

frame. Geometrical constraints like coplanarities and orthogonalities are imposed in the scene. In [16], calibration techniques using similar geometrical constraints are reported. However their method needs user interaction to identify planar surfaces. In our study the detection of planar surfaces is performed automatically during calibration. Once the calibration is performed, a 3D model can be reconstructed from the point data acquired simultaneously using the two laser stripes. In [17] a least squares solution that optimizes linear transformations corresponding to light sources is searched using a complex calibration object that produces 18 distinct point positions. Although their test setup is similar to one of the scanners studied here (Scanner-2 in Fig. 1), the main difference in our approach is that it attempts to generalize double stripe scanning problem of different possible scanning scenarios using a simple calibration object.

The conceptual drawings for the proposed scanners are shown in Fig. 1. The first proposed scanner (i.e. Scanner-1) is an environment scanner with two line lasers. We presented an initial version of this scanner in [18]. The second scanner (i.e. Scanner-2) has the same scanning head (i.e. a camera and two line lasers). The difference from the first one is that Scanner-2 is designed to scan surfaces of near planar objects placed on floor. The third scanner (i.e. Scanner-3) is used to scan objects by using a turntable. The main difference for Scanner-3 is that unlike in the previous scanner models, the camera and lasers are kept stationary in front of a turntable. The object is placed on the turntable and the scanning is performed by turning the turntable by angular increments for $360^{\circ}$.

In this study, the perspective camera model is used. For convenience the axis naming convention in [19] is adopted. As seen in Fig. 2, the image plane is considered to be parallel to the $X Y$ plane where $f$ is the focal distance measured $+Z$ direction.

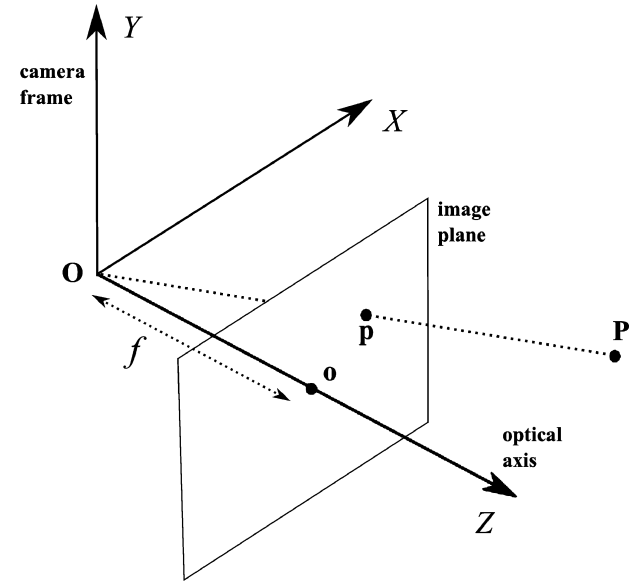

Fig. 2. Perspective camera model as given in [19].

\section{The geometry of double stripe 3D laser scanners}

In this work, we have built three types of scanners with a standard CCD camera and two low cost line lasers. Each one of the scanners is designed to handle a specific need for a 3D scan.

In Scanner-1, the scanning head is placed on a platform which can be controlled by computer and makes "pan" movement (see Fig. 1(a)). Although, the tilt action is also possible on the implemented platform, we only used the pan movements in this study. This radial motion of the platform enables the scanner to scan a surrounding environment. The laser emitters are placed such that they are approximately parallel to each other. 


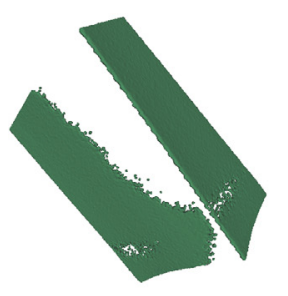

(a) Scan with laser source 1 activated

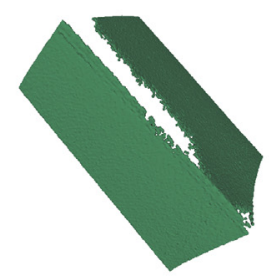

(b) Scan with laser source 2 activated

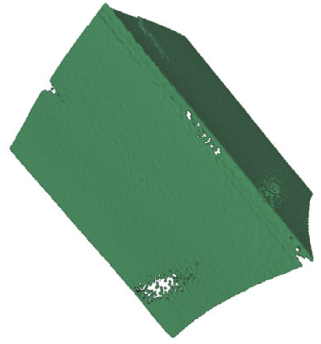

(c) Scan with both laser sources activated

Fig. 3. Usage of two laser sources alleviates the effect of scene occlusions. The calibration block shown in Fig. 9 is used as test object.

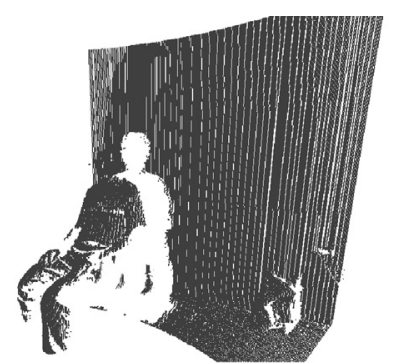

(a)

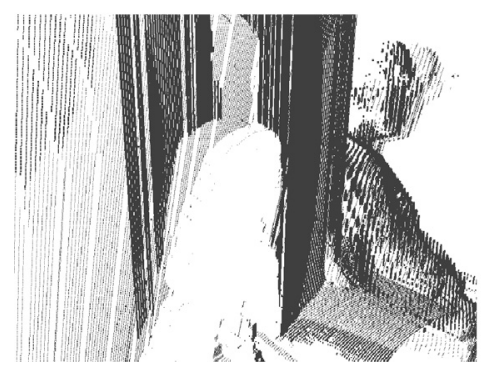

(c)

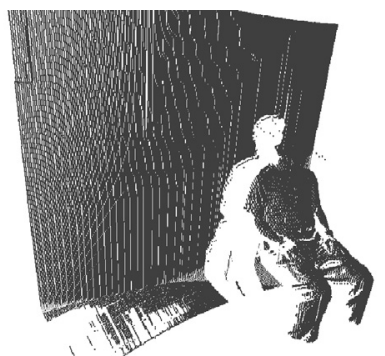

(b)

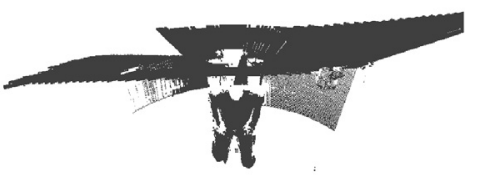

(d)

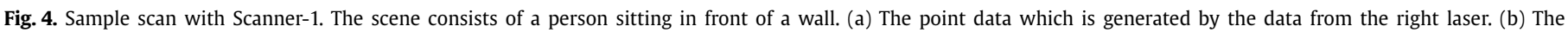
point data which is generated by the data from the left laser. (c) Side view of merged data. (d) Top view of merged data.

Scanner-2 is designed to scan surfaces of objects placed on floor. The scanner formation is depicted in Fig. 1(b). Camera and lasers are attached to a platform which is capable of moving in $X$ axis. This linear motion enables us to scan a surface continuously along $X$ direction.

Lastly, Scanner-3 is designed to scan 3D objects. As in Fig. 1(c), scanning head is fixed. The corresponding object is placed on a turntable. By rotating the object for $360^{\circ}$, object geometry is gathered.

In all three cases, the usage of two line lasers alleviates the effect of scene occlusion. This can be seen in a reconstruction result performed by Scanner-3 in Fig. 3. The scanning results in Figs. 3(a) and 3(b) are individual scans of left and right lasers respectively. Once these results are merged together (see Fig. 3(c)), they complement each other and occlusion effects are significantly eliminated.

\section{Problem statement}

The scanning systems used in this work produce two sets of scanning data which are due to two laser beams. Using two line lasers minimizes the effect of occlusions in the scanning results as shown previously, but it also complicates the calibration of the system. In order to use the scanners we need accurate information about position and orientation of the laser sources to achieve coherent scanning results. In this work, by using known geometrical constraints in the scene, an objective function is defined and Nelder-Mead minimization procedure is used to find the scanner parameters corresponding to a minimum error.

In Fig. 4, scanning results of a person sitting in front of a wall can be seen. This scan data is obtained by using Scanner-1. Fig. 4(a) is the point cloud data which is generated by using the laser source on the left (laser ${ }_{L}$ ). Similarly Fig. 4(b) is generated by the right source $\left(\operatorname{laser}_{R}\right)$. When these scanning results are brought to the same coordinate space it can be seen that the merged sets do not correctly overlap (e.g. Figs. 4(c) and 4(d)). This example shows that using the best-effort manual measurements for the system parameters may provide realistic results for individual laser stripes but the combined data severely suffer from the calibration errors.

\section{Capturing point clouds from double stripes}

\subsection{Camera calibration}

The intrinsic camera calibration is performed as first step. Matlab Toolbox for Camera Calibration [20] which is a MATLAB ${ }^{\mathrm{TM}}$ 


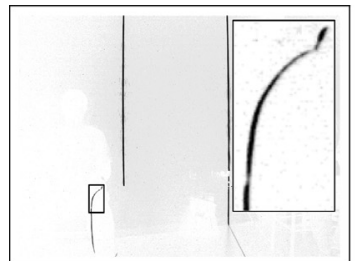

(a)

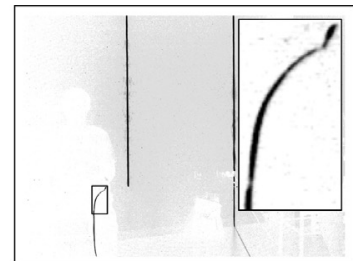

(b)

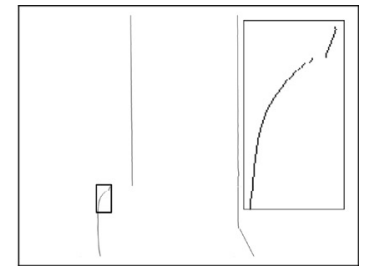

(c)

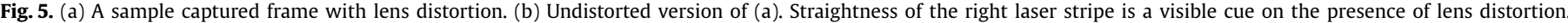

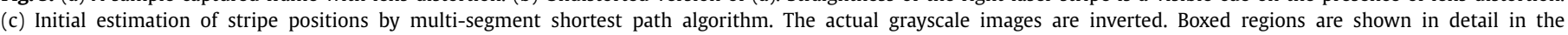
upper-right corner of each figure.

implementation based on [21] is used to find intrinsic camera parameters. These parameters are used in the point cloud generation procedure which is described in the following section.

\subsection{Image pre-processing}

After calibrating intrinsic camera parameters, the image acquisition process is started. The ideal environment is a dark room with surrounding objects. In this scenario the sensor noise becomes significant. To eliminate the sensor noise, median temporal filtering is used. For each stop of the actuators, five consecutive frames are captured and median-filtered. A temporal-filtered frame is captured as in Fig. 5(a) and the undistorted (i.e. corrected from radial distortion using lens calibration results) version can be seen in Fig. 5(b).

\subsection{Path finding in sub-pixel accuracy}

The ambiguities caused by the varying strength of reflected laser light, multiple reflections and independent light sources can be reduced by detecting dominant paths that are most likely due to the reflection of projected stripes. In this work, this is done by using a dynamic programming [22] implementation which computes the top-down shortest path of pixels inversely weighted by their brightness values. The shortest path algorithm is modified to extend the horizontal neighborhood of each pixel using a margin of pixels on each side. The jumps in the stripe reflections due to surface and depth variations are handled by robustly detecting optimal paths in multiple segments. Fig. 5(c) illustrates detected segments in the presence of discontinuities. Besides finding the optimal paths, this algorithm reduces the width of the stripe reflection to a single pixel.

At this stage, the experimental results show that the precision of $3 \mathrm{D}$ points calculated by using the detected path are limited by the resolution of the captured images. The unwanted aliasing effects are clearly visible when smooth planar surfaces are observed (Fig. 6(a)).

To alleviate this problem we regenerated the paths in subpixel accuracy using Nonlinear Least Squares Algorithm (NLSA). As proposed in [23] the width profile of the reflected stripe can be considered as a Gaussian distribution. By fitting a Gaussian function to the laser profile, the approximate peak value can be detected with subpixel accuracy. The optimal paths found by NLSA significantly improve the accuracy of the reconstructed 3D data (e.g. Fig. 6(b)). The details of NLSA implementation are given in Appendix A.

\subsection{Obtaining $3 D$ point cloud}

Considering the assumed perspective camera model, coordinate axes (Fig. 2) and the proposed scanner geometry, the triangula-

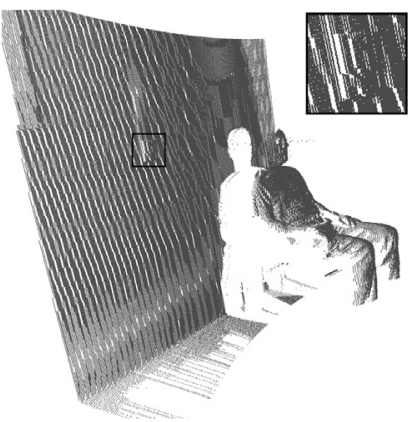

(a)

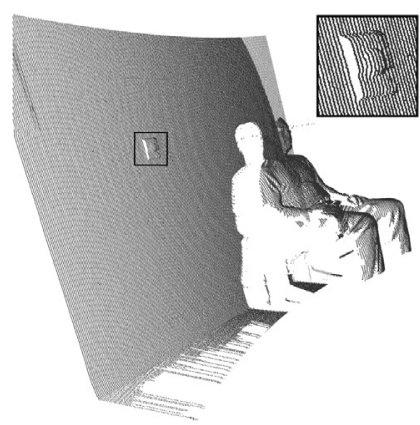

(b)
Fig. 6. (a) Reconstructed point cloud with pixel accuracy. (b) Reconstructed point cloud with sub-pixel accuracy. Boxed regions of both figures are shown in detail in the upper-right corner.

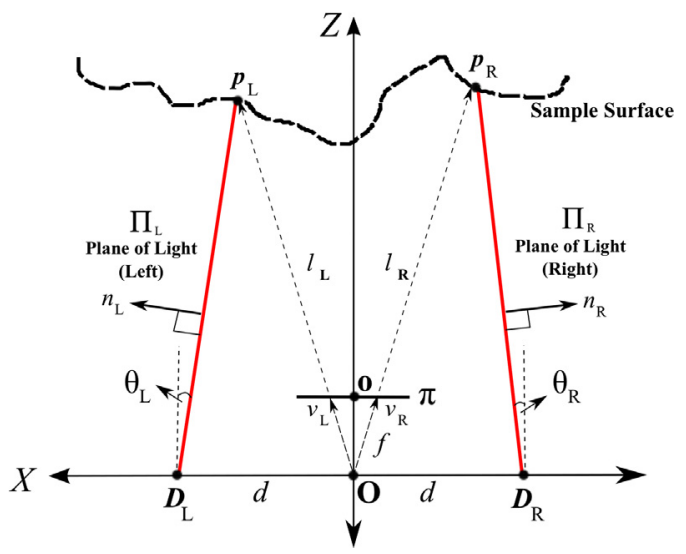

Fig. 7. Laser triangulation geometry for two independent line lasers.

tions can be depicted as Fig. 7. For simplicity, the 2D geometry is shown in $X Z$ plane since the extension to $Y$ axis is straightforward. In this representation, subscript $L$ represents "left" and subscript $R$ represents "right" respectively.

Light plane $\Pi_{L}$ intersects with the objects in the surrounding environment. A point $\mathbf{p}_{L}$ is the intersection point of the plane $\Pi_{L}$ and line $l_{L}$ which means that $\mathbf{p}_{L}$ solves Eqs. (1) and (2) simultaneously. A plane can be defined with one point and one normal vector as in Eq. (1). Similarly, a line can be defined as in Eq. (2).

$\left(\Pi_{L}\right): \quad\left(\mathbf{p}-\mathbf{D}_{L}\right) \cdot \boldsymbol{n}_{L}=0$,

$\left(l_{L}\right): \quad \mathbf{p}=k \boldsymbol{v}_{L}$.

Substituting Eq. (2) in Eq. (1) to find $k$ and substituting this expression back in Eq. (2) the vector $\mathbf{p}_{L}$ pointing to the object surface can be found as: 
$\mathbf{p}_{L}=\left(\frac{\mathbf{D}_{L} \cdot \boldsymbol{n}_{L}}{\boldsymbol{v}_{L} \cdot \boldsymbol{n}_{L}}\right) \boldsymbol{v}_{L}$

In the above derivation, since the distance $d$ (see Fig. 7) is a design parameter by construction, $\mathbf{D}_{L}$ is approximately known. Initially, $n_{L}$ can be considered as the unit vector pointing $+X$ direction. Moreover, $\boldsymbol{v}_{L}$ can be written explicitly as Eq. (4) with $\left(x_{i m}, y_{i m}\right)$ pixel coordinates of the point $\mathbf{p}_{L}$ on the image plane $\pi$, $\left(o_{x}, o_{y}\right)$ the pixel coordinates of the image center, $\left(s_{x}, s_{y}\right)$ the effective size of the pixel in the horizontal and vertical directions and $f$ the focal length of the camera [19]. By writing $\boldsymbol{v}_{L}$ explicitly, the intrinsic parameters of the camera are embedded into the solution (Eq. (5)).

$\begin{aligned} \boldsymbol{v}_{L}= & {\left[\begin{array}{c}-\left(x_{i m}-o_{x}\right) s_{x} \\ -\left(y_{i m}-o_{y}\right) s_{y} \\ f\end{array}\right], } \\ \boldsymbol{p}_{L}= & {\left[\begin{array}{c}-\left(\frac{\mathbf{D}_{L} \cdot \boldsymbol{n}_{L}}{\boldsymbol{v}_{L} \cdot \boldsymbol{n}_{L}}\right)\left(x_{i m}-o_{x}\right) s_{x} \\ -\left(\frac{\mathbf{D}_{L} \cdot \boldsymbol{n}_{L}}{\boldsymbol{v}_{L} \cdot \boldsymbol{n}_{L}}\right)\left(y_{i m}-o_{y}\right) s_{y} \\ \left(\frac{\mathbf{D}_{L} \cdot \boldsymbol{n}_{L}}{\boldsymbol{v}_{L} \cdot \boldsymbol{n}_{L}}\right) f\end{array}\right] . }\end{aligned}$

Similarly $\mathbf{p}_{R}$ can found as in Eq. (6):

$\boldsymbol{p}_{R}=\left[\begin{array}{c}-\left(\frac{\mathbf{D}_{R} \cdot \boldsymbol{n}_{R}}{\boldsymbol{v}_{R} \cdot \boldsymbol{n}_{R}}\right)\left(x_{i m}-o_{x}\right) s_{X} \\ -\left(\frac{\mathbf{D}_{R} \cdot \boldsymbol{n}_{R}}{\boldsymbol{v}_{R} \cdot \boldsymbol{n}_{R}}\right)\left(y_{i m}-o_{y}\right) s_{y} \\ \left(\frac{\mathbf{D}_{R} \cdot \boldsymbol{n}_{R}}{\boldsymbol{v}_{R} \cdot \boldsymbol{n}_{R}}\right) f\end{array}\right]$.

Using Eqs. (5) and (6), and precomputed intrinsic camera parameters (i.e. $o_{x}, o_{y}, s_{x}, s_{y}$ and $f$ ) for projecting image points corresponding to light stripes back to 3D space, point clouds can be created as in Fig. 6.

At each step of the mechanical actuators, an offset (which is angular for Scanners 1 and 3 and translational for Scanner-2) is included in the corresponding terms.

\section{Calibration by using coplanarities and orthogonalities}

\subsection{Geometrical constraints in the scenes}

Previously, details of three scanner configurations were given. They are designed for different scanning scenarios but they share the same calibration problem involving the usage of second line laser. The first scanner, Scanner-1, is designed to scan enclosed environments. A sample scene where a person is sitting in front of a wall can be seen in Fig. 8(a). The most significant geometrical constraint is the wall and the floor data where they represent orthogonal planes. Also it is known that when the best calibration is achieved, corresponding data points from either of the lasers become coplanar on these planes.

The second scanner, Scanner-2, is designed to scan near planar objects. A sample scene in which a Sphinx Mask is laid on the floor can be seen in Fig. 8(b). In this case, there are no apparent geometrical constraints like orthogonal planes in the scene. Also for Scanner-3, if an arbitrary object (e.g. Frog figurine in Fig. 8(c)) is scanned, finding an apparent geometrical constraint is not possible as well. Hence for Scanners 2 and 3 an L-shaped calibration object (see Fig. 9) is utilized to create necessary geometrical constraints in the scene. Once this calibration object is scanned, desired geometrical constraints are obtained. An L-shaped object can also be used for Scanner-1 if two orthogonal planes are not available in the scanned angular interval.

\subsection{Plane detection in $3 D$ point cloud data}

Our solution to the laser calibration problem is to use known orthogonalities and coplanarities in the scene. In [16], the idea of

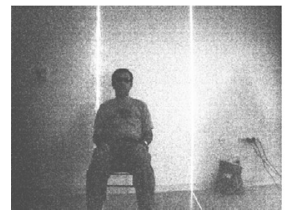

(a) Non-empty room

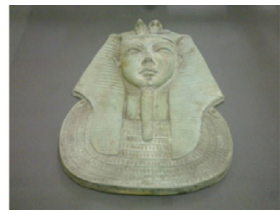

(b) Sphinx mask

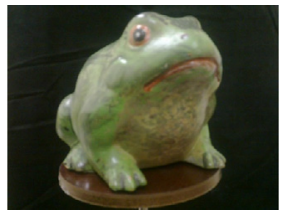

(c) Frog figurine
Fig. 8. Scanned samples.

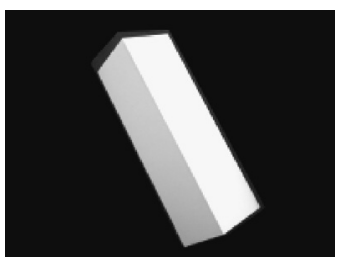

(a) Top view

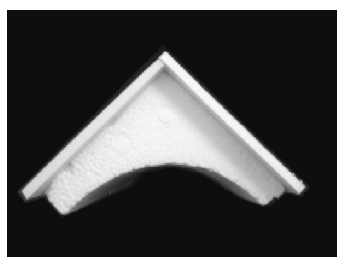

(b) Font view
Fig. 9. L-shaped calibration figure.

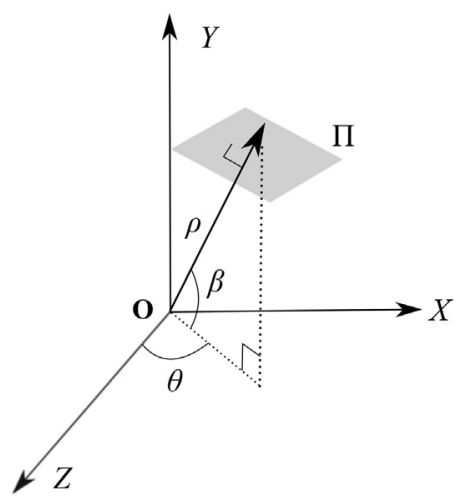

Fig. 10. Parameterization of a plane in 3D.

using orthogonalities and coplanarities was also proposed. In this work the planar features in the scene are found automatically. First, scanning and preliminary 3D reconstruction with initial parameters are performed. Since the number of scanned points is usually extremely high, a brute force method to find dominant planes in 3D data takes an excessive amount of time with conventional computers. Hence an efficient method is needed. In [24], the parameterization of a plane is described as Eq. (7) where $(\rho, \theta, \beta)$ are plane parameters and $(x, y, z)$ triplet is a point on the plane (see Fig. 10).

$\rho=(x \cos (\beta)+y \sin (\beta)) \cos (\theta)+z \sin (\theta)$.

As proposed in [24], plane detection in a point cloud data can be performed by using Hough Transform once the plane is represented with parameters (i.e. $(\rho, \theta, \beta))$. Conventionally, Hough Transform is used to find lines or circles in 2D images. Plane finding in 3D point cloud data is a higher order problem which requires a higher dimensional search. In [25], plane detection methods using Hough Transform are surveyed. Among those methods, Randomized Hough Transform (RHT) is a proper choice for our problem. With a slight modification to the representation in [25], pseudo code of the RHT method we used in our approach can be seen in Appendix B.

When the RHT plane detection algorithm is applied on the point cloud generated using left laser (Fig. 4(b)), the wall data and floor data can be decomposed as Figs. 11(a) and 11(b) respectively. 


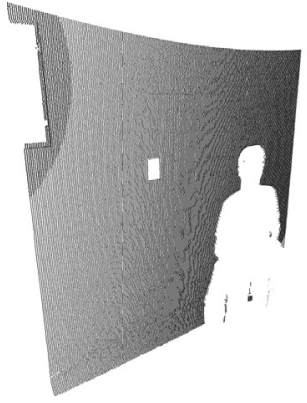

(a)

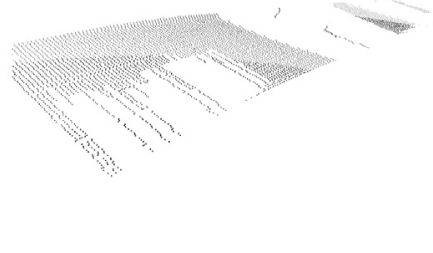

(b)
Fig. 11. (a) Wall data (b) Floor data, detected from point cloud in Fig. 6(b).

Once the (presumably) coplanar points representing a plane are found for both left and right stripes, the corresponding analytical equation can be found by Linear Least Squares Algorithm (LLSA). LLSA based 3D plane fitting allows us to test validity of system parameters so that planes acquired using two stripes are aligned correctly.

To solve 3D plane fitting problem we considered the plane defined in Eq. (8).

$A x+B y+C=z$

In order to find $(A, B, C)$ triplets from observations of $(x, y, z)$ triplets, we focus on the minimization of $\|H u-b\|^{2}$. Here $u=$ $\left[\begin{array}{lll}A & B & C\end{array}\right]^{T}, H$ and $b$ can be constructed from observations. This problem can be solved as in Eq. (9) [26].

$H^{T} H \hat{u}=H^{T} b$.

We can reorganize Eq. (9) as Eq. (10):

$\left[\begin{array}{ccc}\sum_{i=0}^{m} x_{i}^{2} & \sum_{i=0}^{m} x_{i} y_{i} & \sum_{i=0}^{m} x_{i} \\ \sum_{i=0}^{m} x_{i} y_{i} & \sum_{i=0}^{m} y_{i}^{2} & \sum_{i=0}^{m} y_{i} \\ \sum_{i=0}^{m} x_{i} & \sum_{i=0}^{m} y_{i} & \sum_{i=0}^{m} 1\end{array}\right]\left[\begin{array}{c}A \\ B \\ C\end{array}\right]=\left[\begin{array}{c}\sum_{i=0}^{m} x_{i} z_{i} \\ \sum_{i=0}^{m} y_{i} z_{i} \\ \sum_{i=0}^{m} z_{i}\end{array}\right]$.

The solution to Eq. (10) gives us the estimated plane parameters $(A, B, C)$. In our implementation the pixel locations corresponding to dominant planes that are used for calibration are recorded for each image. These image pixels are used in the optimization steps. This makes it possible to complete calibration without imposing extensive memory and computation time requirements.

\subsection{Nelder-Mead Algorithm}

In order to estimate the parameters that represent the projection planes of laser stripes (e.g. $\Pi_{L}$ and $\Pi_{R}$ in Fig. 7), an optimization procedure is required. In our study the problem is defined as a multidimensional minimization problem. Nelder-Mead Algorithm (NMA) [27], which is a downhill simplex method, is an appropriate approach to solve such problems. The problem is solved by calculating objective function $f(\bullet)$ at simplex vertices and performing some procedural geometrical operations to update these vertices. At each step $f(\bullet)$ is calculated for new vertice values. Each vertice corresponds to parameters representing a feasible solution. The details of NMA implementation can be found in Appendix C.

The reconstruction problem for Scanner- 1 and 2 is very similar. The only difference is that: in Scanner- 1 the movement of the scanner is a rotation around $Y$ whereas it is a translation along $X$

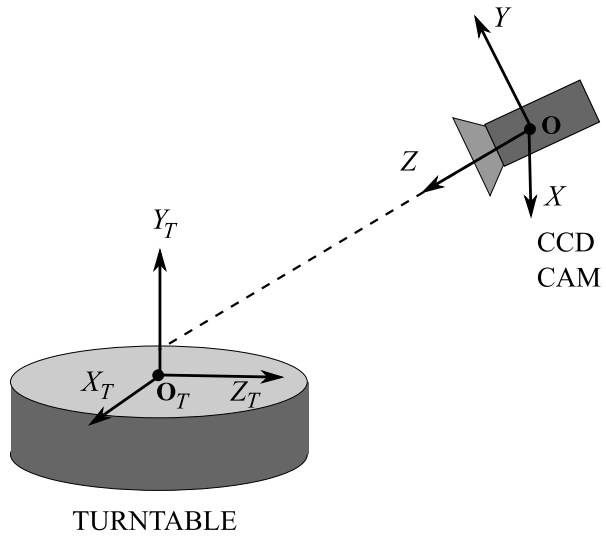

Fig. 12. Axis representations of Scanner-3.

in Scanner-2. The laser projection planes can be identified with $\mathbf{D}_{i}$, $\boldsymbol{n}_{i}$ (where $i=L, R$ ). Surface normals $\boldsymbol{n}_{i}$ can be represented by two angles (i.e. azimuth and zenith angles in spherical coordinates) $\theta_{i}$ and $\beta_{i}$.

The configuration for Scanner-3 (see Fig. 12) is slightly different since the movement is with respect to a turntable. In addition to the configuration parameters given for Scanners 1 and 2, the rotation around turntable's $Y_{T}$ axis should also be embedded in the solution. Coordinates of the turntable's rotation center (i.e. $\boldsymbol{O}_{T}$ ) should be expressed with respect to cameras optical center (i.e. $\mathbf{O}$ ). The position of the turntable is included in the optimization procedure with three parameters $D_{x}, D_{y}$ and $D_{z}$. Initial distance values can be approximately found by measuring the distances manually. In order to correctly identify the rotation around turntable axis, the necessary rotation angles $\Theta_{\chi}$, $\Theta_{y}$ and $\Theta_{z}$, which aligns corresponding coordinate axes, are also included in the optimization procedure. The laser emitters can be positioned such that the stripes intersect behind the rotation center. This offset distance is dependent on the size and shape of the object that needs to be scanned. It is possible to scan non-convex objects if a significant offset which should be lower than approximate radius of the object is used (e.g. Fig. 13). In Fig. 13(a) the laser stripes are intersected at the rotation axis of the turntable. Since some part of the non-convex sample is located beyond the intersection point, an ambiguity is observed while generating point clouds by using Eqs. (5) and (6). This ambiguity is resolved by intersecting lasers beyond the rotation axis (Fig. 13(b)).

\subsection{Objective function}

In order to make 3D measurements from left and right stripes align correctly, the parameters that represent laser sources' positions and orientations should be identified accurately. These parameters are listed in Eq. (11). Here, $\boldsymbol{S}_{1}, \boldsymbol{S}_{2}$ and $\boldsymbol{S}_{3}$ are the parameter sets used for Scanners 1, 2 and 3 respectively.

The geometrical constraints of the calibration problem can be illustrated as in Fig. 14. The planar point clouds are named as $\pi_{i}^{j}$ where subscript $i$ represents laser source (i.e. either left or right) and superscript $j$ represents the corresponding surface (i.e. either $a$ or $b$ ) of the scanned environment or the calibration pattern. After optimizing the scanner parameters, $\pi_{L}^{a}$ and $\pi_{R}^{a}$ become coplanar in $\Pi^{a}$. Similarly $\pi_{L}^{b}$ and $\pi_{R}^{b}$ become coplanar in $\Pi^{b}$.

To measure the coplanarity we utilized linear-least squares method to find the best fitting plane equations for $\Pi^{j} \mathrm{~s}$ which represent the merged plane pairs. The fitting error (i.e. $\left.\operatorname{Err}\left(\Pi^{j}\right)\right)$ 


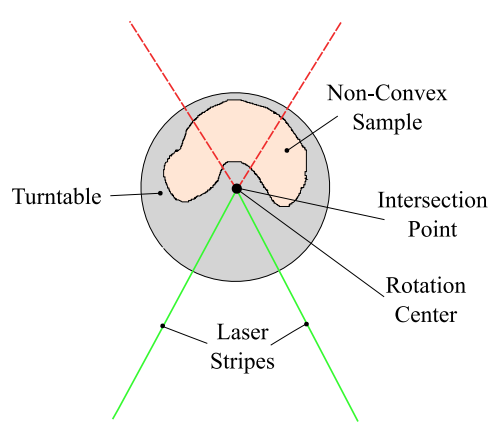

(a) Lasers are intersected at turntable rotation axis.

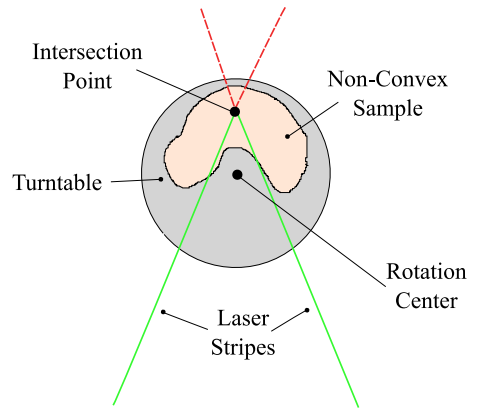

(b) Lasers are intersected beyond turntable rotation axis.

Fig. 13. Scanning non-convex objects using Scanner-3.

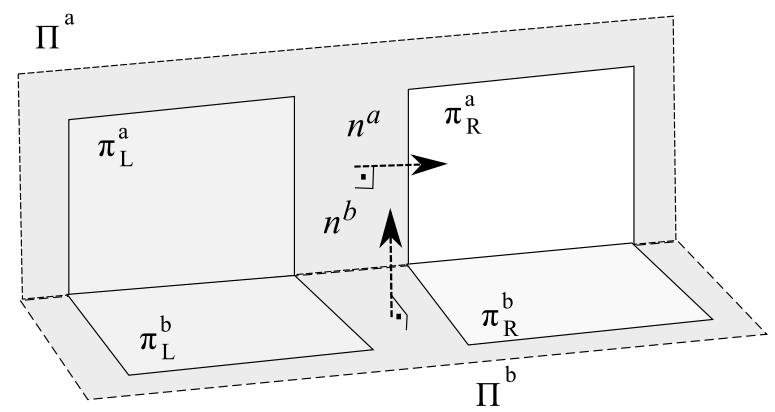

Fig. 14. Representation of the geometry used to define objective function in NMA.

serves as a measure that quantifies coplanarity of two planes (i.e. $\pi_{L}^{j}$ and $\pi_{R}^{j}$ ). The orthogonality constraint is included in the objective function as the dot product of normals $n^{a}, n^{b}$ (of surfaces $\Pi^{a}$ and $\left.\Pi^{b}\right)$.

Scanning results are significantly improved after NMA iterations on the objective function (Figs. 18, 19, 20, 21).

$$
\begin{aligned}
& \mathbb{S}_{1}=\left\{\theta_{L}, \beta_{L}, \theta_{R}, \beta_{R}, D_{L}, D_{R}\right\}, \\
& \mathbb{S}_{2}=\left\{\theta_{L}, \beta_{L}, \theta_{R}, \beta_{R}, D_{L}, D_{R}\right\}, \\
& \mathbb{S}_{3}=\left\{\theta_{L}, \beta_{L}, \theta_{R}, \beta_{R}, D_{L}, D_{R}, D_{x}, D_{y}, D_{z}, \Theta_{x}, \Theta_{y}, \Theta_{z}\right\} . \\
& f\left(\mathbb{S}_{i}\right)=\operatorname{MAX}\left\{\operatorname{Err}\left(\Pi^{a}\right), \operatorname{Err}\left(\Pi^{b}\right)\right\} *\left(1+\left(n^{a} \cdot n^{b}\right)\right), \\
& \quad i=1,2,3 .
\end{aligned}
$$

\section{Results and conclusion}

In this study three different laser scanners are studied. Each scanner has two line lasers installed at each side of a standard CCD camera (see Fig. 1). Using two laser stripes brings up the problem of aligning two scanning results gathered from each one of the laser sources. To correctly align scanning results, both laser light planes should be correctly identified with respect to a reference coordinate frame which is the camera coordinate system described in Fig. 2.

In the previous sections, the problem is defined and geometrically parameterized. It is shown that parameters can be optimized by a minimization procedure. NMA is chosen for this multidimensional minimization problem. Sample optimization results can be seen in Table 1. For calibration of Scanner-1, wall and floor data from the room scan is used. For Scanners 2 and 3, scanning result of the calibration object (see Fig. 9) is used. Angles are given in degrees and distances are given in millimeters

\begin{tabular}{|c|c|c|c|c|}
\hline \multicolumn{2}{|c|}{ Parameter \Scanner } & \multirow{2}{*}{$\begin{array}{r}\text { Scanner-1 } \\
0.000\end{array}$} & \multirow{2}{*}{$\begin{array}{r}\text { Scanner-2 } \\
36.000\end{array}$} & \multirow{2}{*}{$\begin{array}{r}\text { Scanner-3 } \\
15.000\end{array}$} \\
\hline$\theta_{L}$ & initial & & & \\
\hline & final & 0.211 & 37.130 & 14.456 \\
\hline \multirow{2}{*}{$\beta_{L}$} & initial & 0.000 & 0.000 & 0.000 \\
\hline & final & -0.003 & 0.332 & -4.012 \\
\hline \multirow[t]{2}{*}{$\theta_{R}$} & initial & 180.000 & 145.000 & 160.000 \\
\hline & final & 179.787 & 143.633 & 160.898 \\
\hline \multirow[t]{2}{*}{$\beta_{R}$} & initial & 0.000 & 0.000 & 0.0 \\
\hline & final & 0.841 & -0.295 & -5.531 \\
\hline \multirow[t]{2}{*}{$D_{L}$} & initial & 500.000 & 250.000 & 137.700 \\
\hline & final & 502.950 & 251.913 & 134.489 \\
\hline \multirow[t]{2}{*}{$D_{R}$} & initial & -500.000 & -250.000 & -137.700 \\
\hline & final & -502.574 & -247.474 & -138.033 \\
\hline \multirow[t]{2}{*}{$D_{x}$} & initial & $\mathrm{N} / \mathrm{A}$ & $\mathrm{N} / \mathrm{A}$ & 0.000 \\
\hline & final & & & 19.692 \\
\hline \multirow[t]{2}{*}{$D_{y}$} & initial & $\mathrm{N} / \mathrm{A}$ & $\mathrm{N} / \mathrm{A}$ & -92.100 \\
\hline & final & & & -96.645 \\
\hline \multirow[t]{2}{*}{$D_{z}$} & initial & $\mathrm{N} / \mathrm{A}$ & $\mathrm{N} / \mathrm{A}$ & 448.400 \\
\hline & final & & & 439.066 \\
\hline \multirow[t]{2}{*}{$\Theta_{x}$} & initial & $\mathrm{N} / \mathrm{A}$ & $\mathrm{N} / \mathrm{A}$ & 22.600 \\
\hline & final & & & 17.553 \\
\hline \multirow[t]{2}{*}{$\Theta_{y}$} & initial & $\mathrm{N} / \mathrm{A}$ & $\mathrm{N} / \mathrm{A}$ & 0.000 \\
\hline & final & & & -24.126 \\
\hline \multirow[t]{2}{*}{$\Theta_{z}$} & initial & $\mathrm{N} / \mathrm{A}$ & $\mathrm{N} / \mathrm{A}$ & 0.000 \\
\hline & final & & & -1.883 \\
\hline \multirow[t]{2}{*}{$f(\bullet)$} & initial & 22.166 & 3.332 & 1.241 \\
\hline & final & 0.074 & 0.482 & 0.147 \\
\hline
\end{tabular}
(mm).
Table 1

Sample optimization results for parameters. Angle values are given in degrees, distances are given in millimeters (mm).

To visualize the performance of the optimization procedure, parameter variations during NMA iterations are given in Fig. 15. Since the parameters are in different metrics they are normalized with respect to the maximum value achieved during iterations.

Variations in objective function value during NMA iterations are given in Fig. 16. The algorithm is stopped at a point where the parameter and objective function variations are negligibly small.

Reconstructed scanning results can be seen in Figs. 18-20. To reconstruct surfaces a distance function is calculated over a computation grid [28,29] and triangulated by using Marching Cubes Algorithm (MCA) [30].

The total computation time on a set of $2501280 \times 1024$ images using a Core i5 $2.53 \mathrm{GHz}$ based computer is typically less than 5 minutes. The most significant computationally inten- 

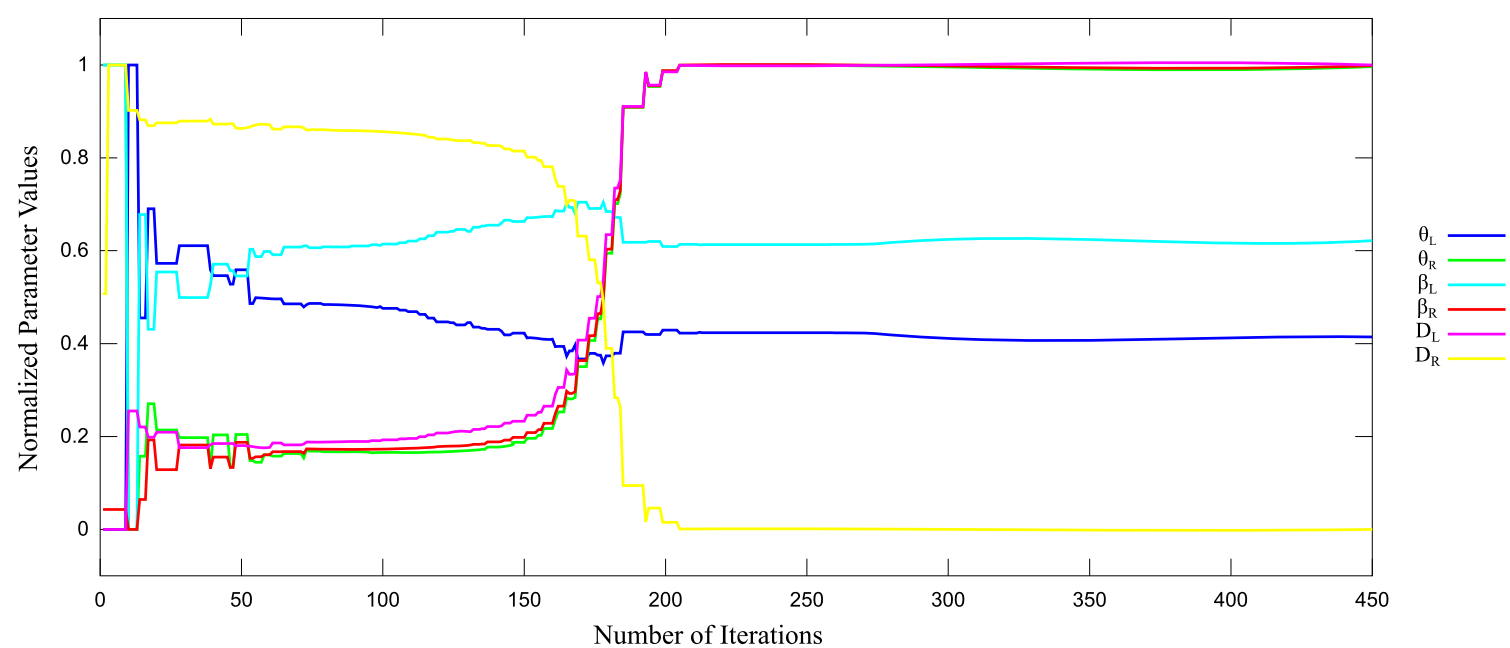

(a) Scanner-1

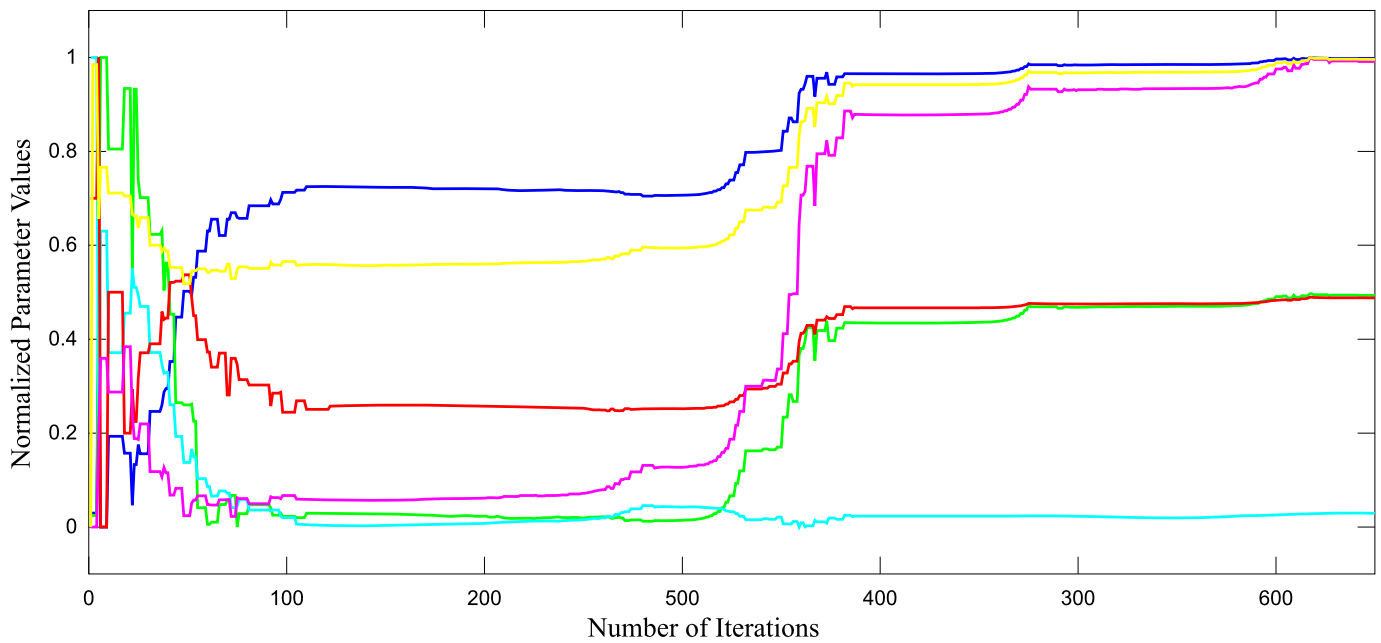

$\theta_{\mathrm{L}}$
$\theta_{\mathrm{R}}$
$\beta_{\mathrm{L}}$
$\beta_{\mathrm{R}}$
$\mathrm{D}_{\mathrm{L}}$
$\mathrm{D}_{\mathrm{R}}$

(b) Scanner-2
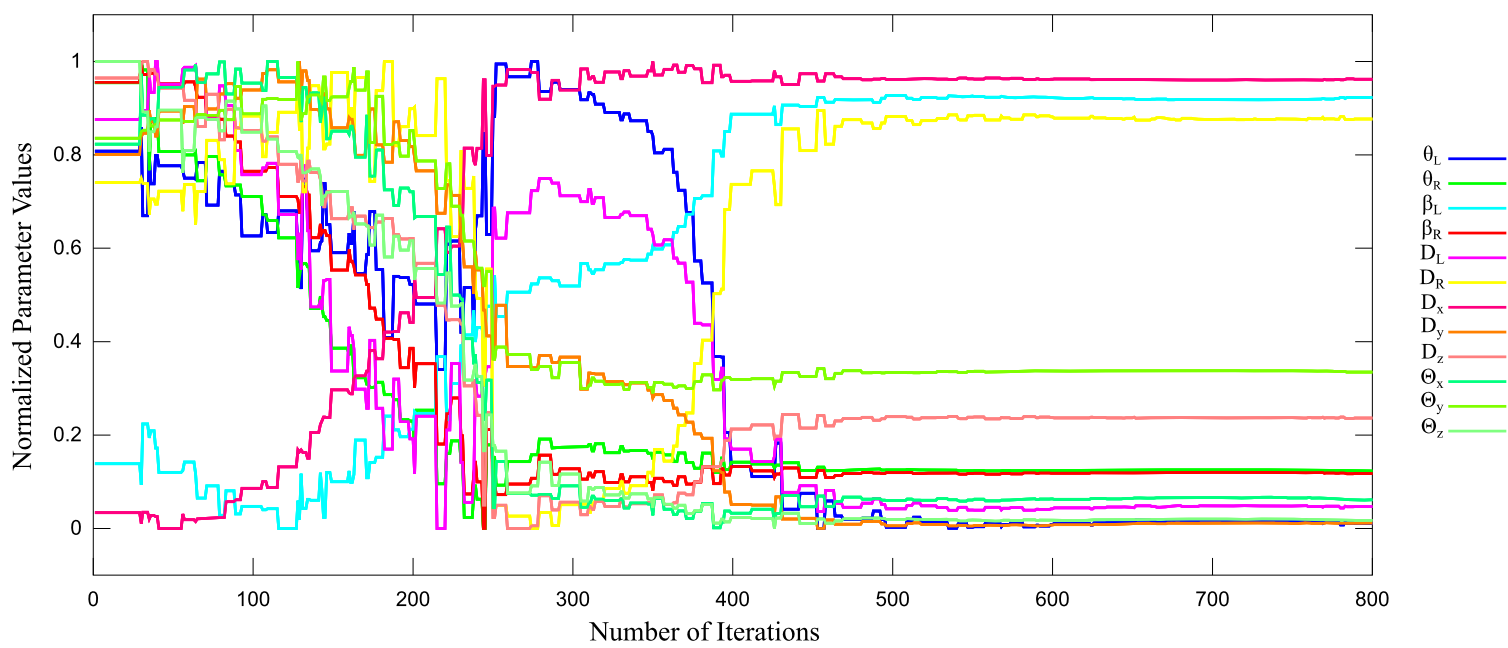

(c) Scanner-3

Fig. 15. Parameter changes during NMA iterations. 


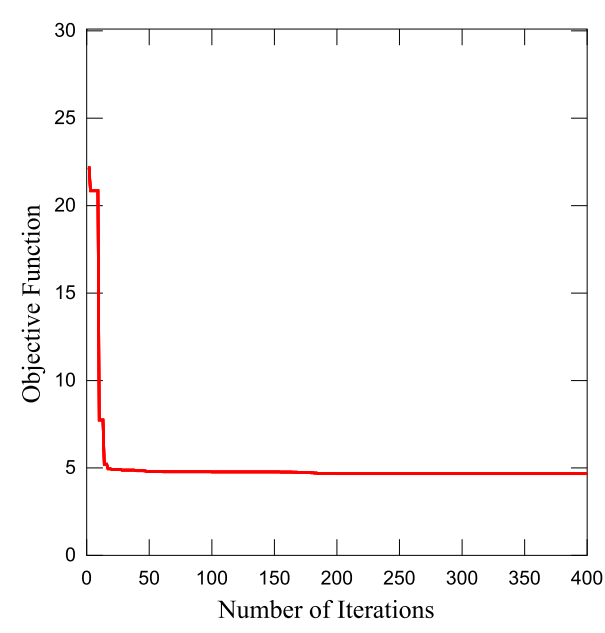

(a) Scanner-1

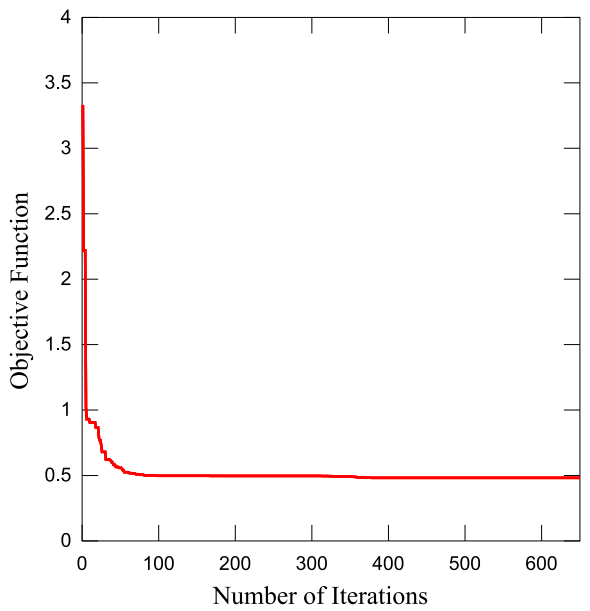

(b) Scanner-2

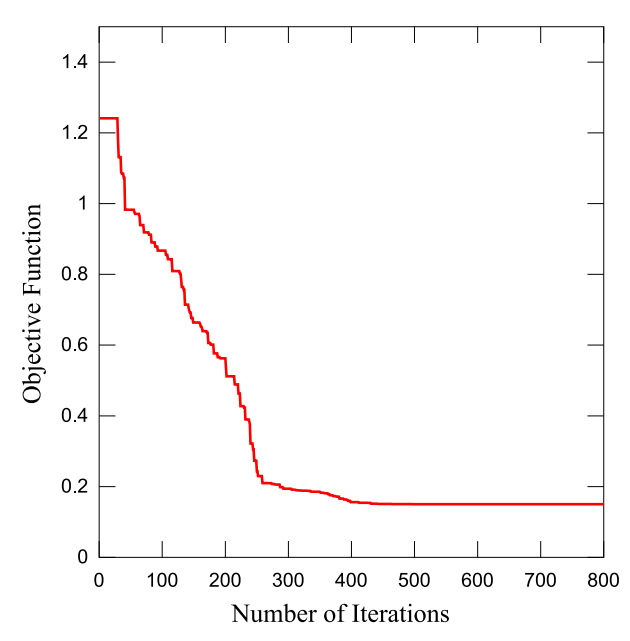

(c) Scanner-3

Fig. 16. Value of objective function (see Eq. (12)) during NMA iterations.

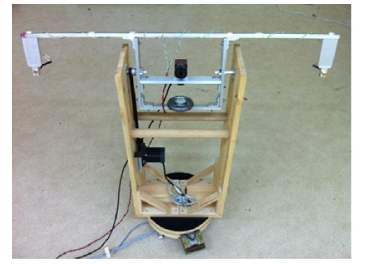

(a) Scanner-1

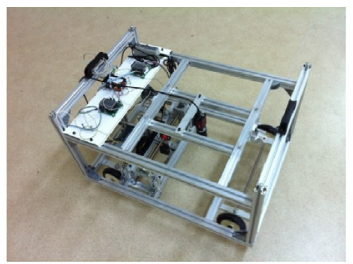

(b) Scanner-2

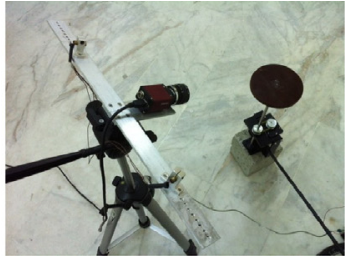

(c) Scanner-3

Fig. 17. Images of the actual scanners developed for this study.

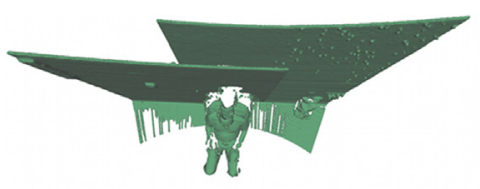

(a) Top view before optimization

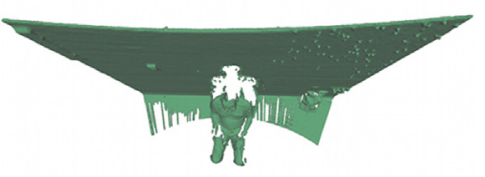

(b) Top view after optimization

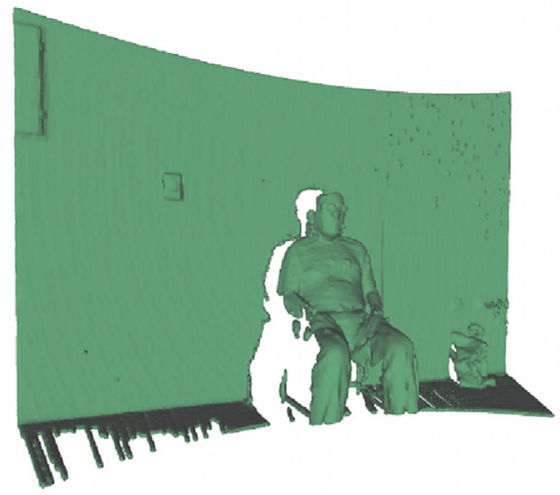

(c) Front view after optimization

Fig. 18. Reconstruction results for "person sitting in front of a wall" scan by Scanner-1. 


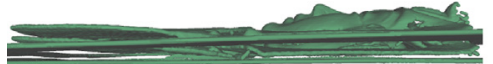

(a) Side view before optimization

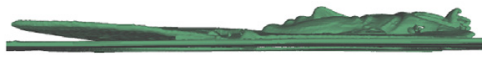

(b) Side view after optimization

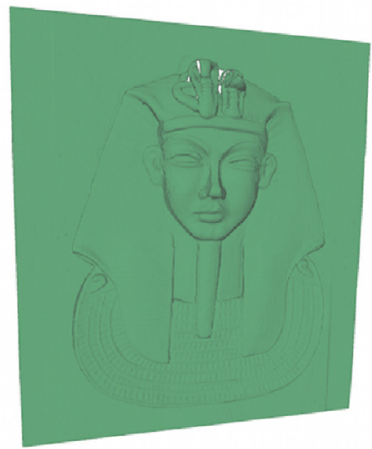

(c) Front view after optimization

Fig. 19. Reconstruction results for "Sphinx Mask" scan by Scanner-2.

Table 2

Mean and standard deviations of radius measurements for a sphere (shown in Fig. 21) using left and right lasers before and after optimization procedure.

\begin{tabular}{llll}
\hline (Before optim./after optim.) & Left laser & Right laser & Left and right laser \\
\hline Mean & $99.528 / \mathbf{7 5 . 3 9 9}$ & $122.610 / \mathbf{7 4 . 9 8 9}$ & $112.220 / \mathbf{7 5 . 1 7 2}$ \\
Standard deviation & $6.257 / \mathbf{0 . 5 0 8}$ & $4.920 / \mathbf{0 . 5 3 8}$ & $12.755 / \mathbf{0 . 5 6 2}$ \\
Number of data points & $90000 / \mathbf{9 0 0 0 0}$ & $90000 / \mathbf{9 0 0 0 0}$ & $90000 / \mathbf{9 0 0 0 0}$ \\
\hline
\end{tabular}

sive operations are done on RHT and NMA. Detection of four dominant planes using RHT for Scanner-3 setup is completed in 62 seconds. NMA (whose performance is highly dependent on initial parameter assignments) for the same setup is completed in 192 seconds. Scanner-1 and Scanner-2 computations are completed in a shorter period due to less number of parameters, even though twice as many (500) image frames are used.

A final test is done to test the validity of the procedure using an object with known geometrical properties. In order to generate worst case scenario, Scanner-3 is used in this experiment, since it involves additional parameters compared to other scanners. A sphere with a diameter of $150 \mathrm{~mm}$ was chosen as the test object. The point clouds are shown before and after optimization in parts (a) and (b) of Fig. 21. Parts (c) and (d) of the same figure show the histograms of distances from detected sphere center to 3D points. Same number of data points are randomly selected for before and after optimization cases. Numerical results from this experiment are given in Table 2. It can be seen that, refinement in the estimated parameter set makes it possible to generate $3 \mathrm{D}$ points preserving the shape of the object. In this experiment the size of the object is correctly identified, but it should be noted that the distance measurements are dependent mostly on the parameters $D_{L}$ and $D_{R}$ which are the distances from camera to laser sources (see Fig. 7). The alignment of point sets does not guarantee convergence to physically correct values. To determine the correct scales of $D_{L}$ and $D_{R}$, precise physical measurements of these terms can be used as fixed values or a calibration object with known geometry can be utilized.

In this paper, it is shown that the usage of double laser stripes effectively alleviates the occlusion problems. The problems associated with multiple stripes are solved using an optimization procedure which calibrates system parameters. Calibration performance is illustrated for three different types of scanning systems

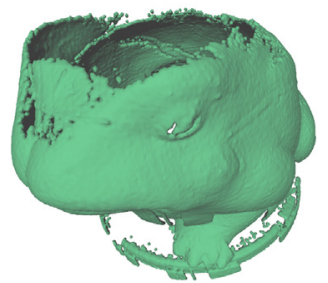

(a) Front view before optimization

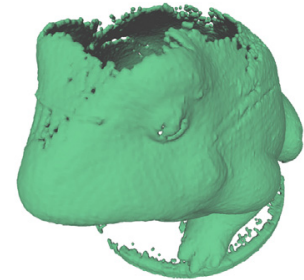

(b) Front view after optimization
Fig. 20. Reconstruction results for "Frog figurine" scan by Scanner-3.

(Fig. 17) designed for indoor environments, near planar objects and small 3D objects. The calibration object needed for our procedure is a simple object that has two perpendicular planar surfaces. It is also shown that in some cases where such surfaces exist in the scene, calibration can be done without using an external calibration object.

\section{Acknowledgments}

We wish to thank the anonymous reviewers who helped us improve this paper with their valuable comments and suggestions. We also would like to acknowledge Burak Çalargün and Çag̃rı Pala for their contributions during the construction of the scanners.

\section{Appendix A. NLSA for Gaussian fit}

$f(x ; \mathbf{a})=a_{1} e^{-\left(x-a_{2}\right) / 2 a_{3}^{2}}$.

To obtain a subpixel accuracy Gaussian function can be fitted to the laser profile. In Eq. (A.1), $a_{1}$ is the amplitude of the Gaus- 


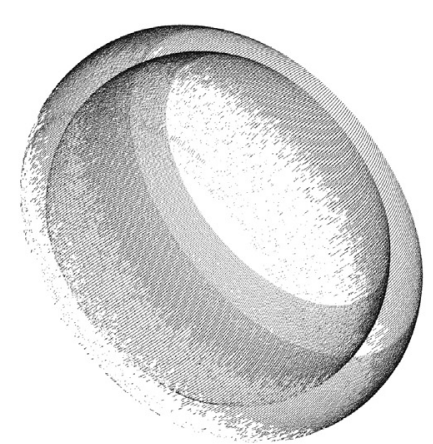

(a)

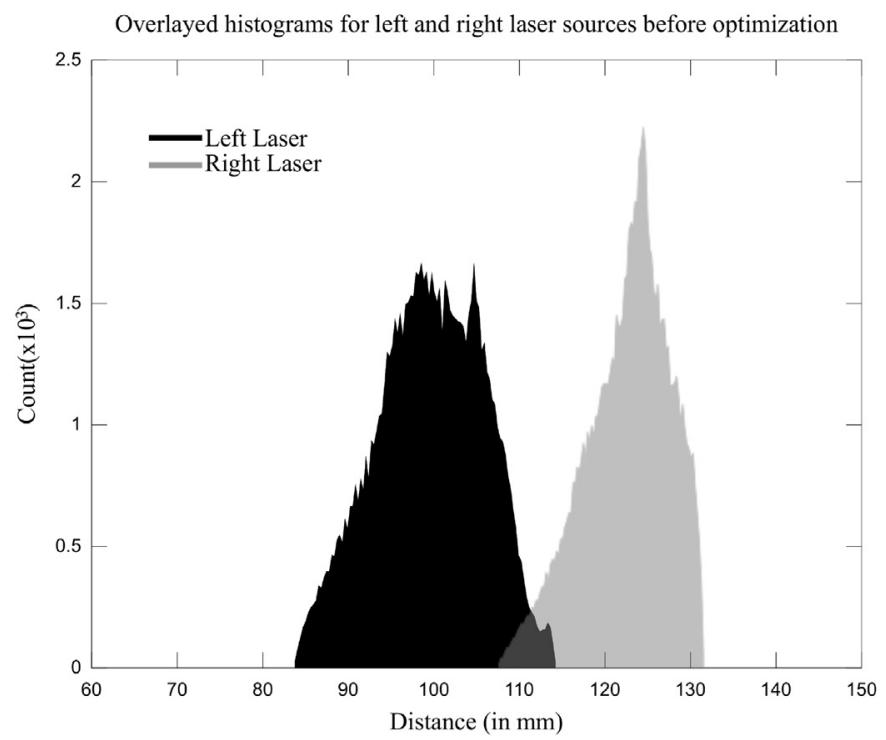

(c)

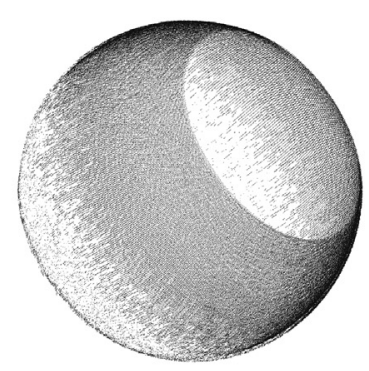

(b)

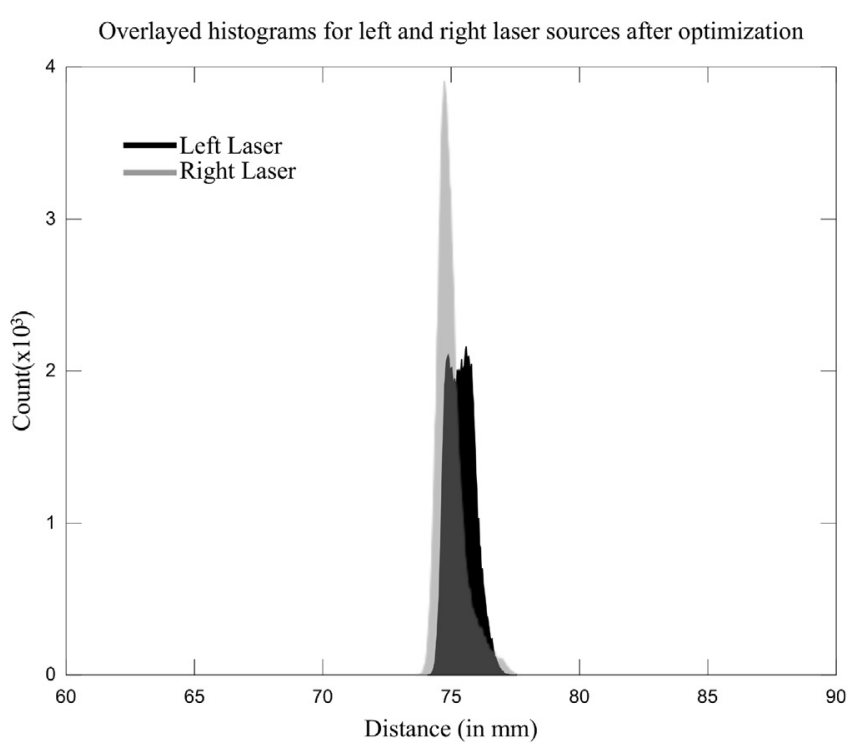

(d)

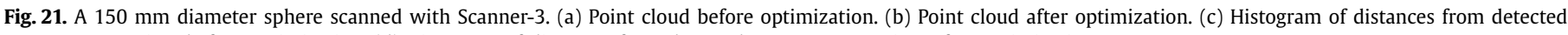
center to 3D points before optimization. (d) Histogram of distances from detected center to 3D points after optimization.

sian which in our problem corresponds to the pixel intensity. $a_{2}$ is the position of the peak point, which is the value we aimed to find and lastly $a_{3}$ corresponds to the standard deviation which identifies the width of the bell shape of the Gaussian. Nonlinear least-squares algorithm [31] is implemented as the following to solve this problem:

minimize $\left(\sum_{i=1}^{m}\left(r_{i}\left(x_{i} ; \mathbf{a}\right)\right)^{2}\right)$,

where $m$ is the number of measurements and $r_{i}$ can be defined as the difference between $i$ th measured value $y_{i}$ and the fitted function value $f\left(x_{i}, \mathbf{y}\right)$. Hence $r_{i}$ can be written as:

$r_{i}\left(x_{i}, \mathbf{a}\right)=y_{i}-f\left(x_{i} ; a_{1}, a_{2}, \ldots, a_{n}\right)$.

In our problem, the number of variables $n$ is 3 (considering Eq. (A.1)). $\mathbf{r}$ can be considered a small change in the function $f(x ; \mathbf{a})$. Hence the linearized estimate for the small changes in the function can also be written as (A.4).

$r_{i}\left(x_{i}, \mathbf{a}\right)=\left.\sum_{j=1}^{n} \frac{\partial f}{\partial a_{j}} d a_{j}\right|_{x_{i} ; \mathbf{a}}$.
After constructing an $m \times n$ Jacobian matrix $\mathbf{J}$ from the terms:

$J_{i j}=\left.\frac{\partial f}{\partial a_{j}}\right|_{x_{i} ; \mathbf{a}}$

the displacement vector $d \mathbf{a}$ can be found as:

$d \mathbf{a}=\left(\mathbf{J}^{T} \mathbf{J}\right)^{-1}(\mathbf{J} \mathbf{r})$

In our problem, $m=9$, i.e. the Gaussian fit is performed by using nine neighboring pixels in the same row, where the mid pixel is the pixel found by dynamic programming. The original pixel intensity value at the coordinate where we find a path pixel in the binary path image is taken as initial $a_{1}$, the column position is taken as initial $a_{2}$ and initial $a_{3}$ is taken as 2.0 (in Eq. (A.1)). $y_{i}$ values are taken as corresponding nine neighboring pixels in the original image. da value is used to find the $a_{j}$ estimates in an iterative manner. At each iteration small $d a_{j}$ variations are added to each $a_{j}$ and by updating $\mathbf{r}$ and $\mathbf{J}$ at each iteration step optimal values are achieved after few iterations. Pixel accurate and subpixel accurate reconstruction results can be seen in Figs. 6(a) and 6 (b) respectively. 


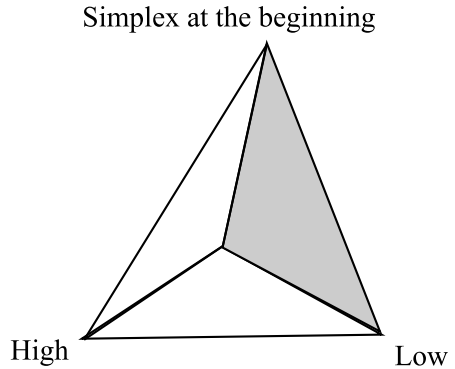

(a)

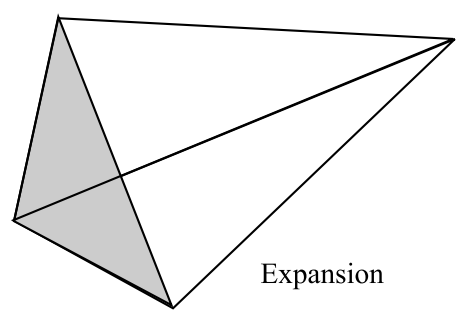

(c)

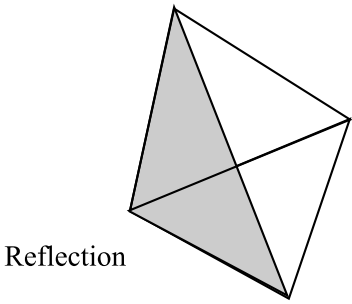

(b)

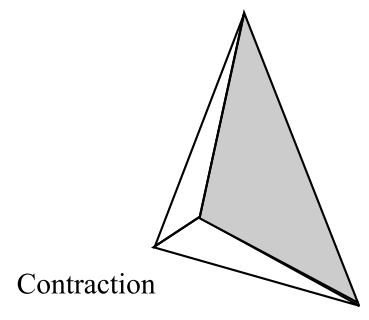

(d)

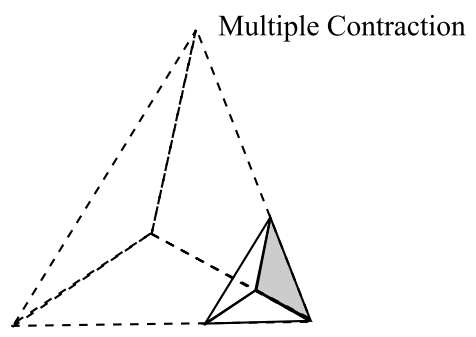

(e)

Fig. C.1. Actions in Nelder-Mead Algorithm.

\section{Appendix B. Randomized Hough Transform (RHT)}

Randomized Hough Transform (RHT)

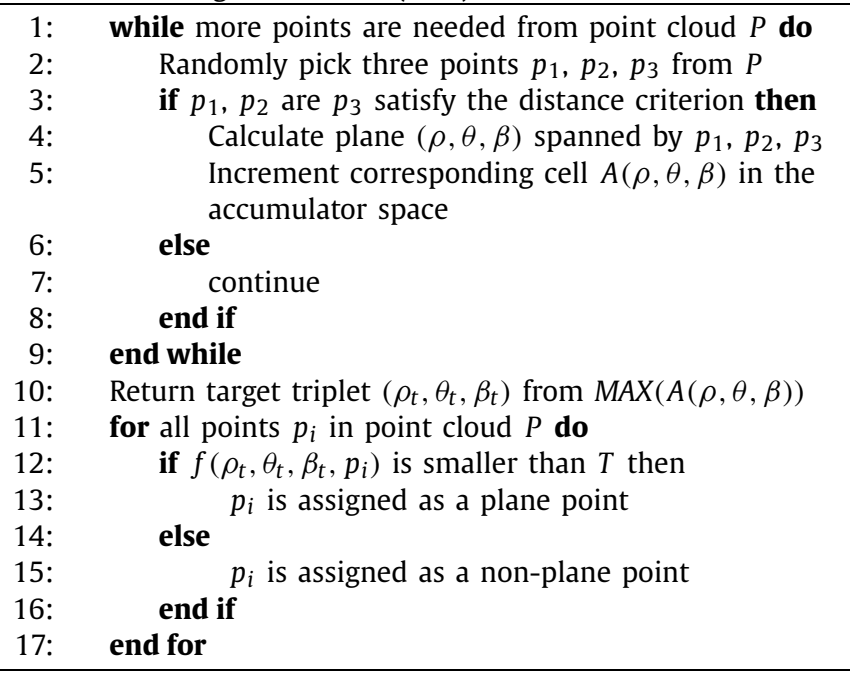

In the above code, $P$ represents the corresponding point cloud in which a plane is searched for. The key idea of RHT is to randomly pick point triplets (i.e. $\left.\left(p_{1}, p_{2}, p_{3}\right)\right)$ and parameterize the plane which is defined by the three chosen points. The distribution of plane parameter triplets (i.e. $(\rho, \theta, \beta)$, see Fig. 10) concentrates around a point (i.e. $\left.\left(\rho_{t}, \theta_{t}, \beta_{t}\right)\right)$ in parameter space and this point represents a plane equation. Once the parameters are found, the last step is to find the points which are close enough to the plane defined in Eq. (7).

\section{Appendix C. Nelder-Mead Algorithm (NMA)}

The NMA can be described as follows:

Nelder-Mead Algorithm

\section{STEP 1: Initialize:}

Order vertices according to the objective function values (Fig. C.1(a)).

\section{STEP 2: Calculate COG:}

$$
f\left(\mathbf{x}_{1}\right) \leqslant f\left(\mathbf{x}_{\mathbf{2}}\right) \leqslant \cdots \leqslant f\left(\mathbf{x}_{\mathbf{N}+\mathbf{1}}\right)
$$

$\mathbf{x}_{\mathbf{0}}$ which is the center of gravity of $\left(\mathbf{x}_{1}, \mathbf{x}_{2}, \ldots, \mathbf{x}_{\mathrm{N}}\right)$ excluding $\mathbf{x}_{\mathbf{N}+\mathbf{1}}$

\section{STEP 3: Reflection:}

Compute reflected point $\mathbf{x}_{\mathbf{R}}=\mathbf{x}_{\mathbf{0}}+\alpha\left(\mathbf{x}_{\mathbf{0}}-\mathbf{x}_{\mathbf{N}+\mathbf{1}}\right)$

(Fig. C.1(b))

If $f\left(\mathbf{x}_{\mathbf{1}}\right) \leqslant f\left(\mathbf{x}_{\mathbf{R}}\right) \leqslant f\left(\mathbf{x}_{\mathbf{N}}\right)$

Then replace $\mathbf{x}_{\mathbf{N}+1}$ with $\mathbf{x}_{\mathbf{R}}$

STEP 4: Expansion:

If $\mathbf{x}_{\mathbf{R}}<\mathbf{x}_{1}$

Then compute expanded point $\mathbf{x}_{\mathbf{E}}=\mathbf{x}_{\mathbf{0}}+\gamma\left(\mathbf{x}_{\mathbf{o}}-\mathbf{x}_{\mathbf{N}+\mathbf{1}}\right)$ (Fig. C.1(c))

$$
\text { If } \mathbf{x}_{\mathbf{E}}<\mathbf{x}_{\mathbf{R}}
$$

Then replace $\mathbf{x}_{\mathbf{N}+1}$ with $\mathbf{x}_{\mathbf{E}}$ and goto STEP 1

Else replace $\mathbf{x}_{\mathbf{N}+1}$ with $\mathbf{x}_{\mathbf{R}}$ and goto STEP 1

\section{STEP 5: Contraction:}

\section{Since $\mathbf{x}_{\mathbf{R}} \geqslant \mathbf{x}_{\mathbf{N}}$}

Compute contracted point $\mathbf{x}_{\mathbf{C}}=\mathbf{x}_{\mathbf{N}+\mathbf{1}}+\rho\left(\mathbf{x}_{\mathbf{0}}-\mathbf{x}_{\mathbf{N}+\mathbf{1}}\right)$

(Fig. C.1(d))

If $\mathbf{x}_{\mathbf{C}}<\mathbf{x}_{\mathbf{N}+\mathbf{1}}$

Then replace $\mathbf{x}_{\mathbf{N}+\mathbf{1}}$ with $\mathbf{x}_{\mathbf{C}}$ and goto STEP 1

Else goto STEP 6

STEP 6: Multiple Contraction:

Compute contracted points $\mathbf{x}_{\mathbf{i}}=\mathbf{x}_{\mathbf{1}}+\sigma\left(\mathbf{x}_{\mathbf{i}}-\mathbf{x}_{\mathbf{1}}\right)$

For $i \in\{2, \ldots, N+1\}$ (Fig. C.1(e))

Else goto STEP 1

In the above algorithm, $\mathbf{x}$ represents optimization parameters given as in Eq. (11) for each scanner.

\section{References}

[1] D. Scharstein, R. Szeliski, A taxonomy and evaluation of dense two-frame stereo correspondence algorithms, Int. J. Comput. Vis. 47 (2002) 7-42.

[2] B.K.P. Horn, Robot Vision, MIT Electrical Engineering and Computer Science Series, MIT Press, 1986

[3] R.J. Woodham, Shape recovery, in: L.B. Wolff, S.A. Shafer, G.E. Healey (Eds.), Shape Recovery, Jones and Bartlett Publishers, Inc., USA, 1992, pp. 115-120, (Ch. Photometric method for determining surface orientation from multiple images).

[4] J. Ens, P. Lawrence, An investigation of methods for determining depth from focus, IEEE Trans. Pattern Anal. Mach. Intell. 15 (2) (1993) 97-108.

[5] M. Subbarao, G. Surya, Depth from defocus: A spatial domain approach, Int. J. Comput. Vis. 13 (1994) 271-294

[6] M. Watanabe, S.K. Nayar, Rational filters for passive depth from defocus, Int. J Comput. Vis. 27 (1998) 203-225.

[7] G.A. Atkinson, E. Hancock, Shape estimation using polarization and shading from two views, IEEE Trans. Pattern Anal. Mach. Intell. 29 (2007) 2001-2017.

[8] S. Rahmann, N. Canterakis, Reconstruction of specular surfaces using polarization imaging, Proc. IEEE Comput. Soc. Conf. Comput. Vis. Pattern Recognit. 1 (2001) 149-155.

[9] J. Salvi, S. Fernandez, T. Pribanic, X. Llado, A state of the art in structured light patterns for surface profilometry, Pattern Recognit. 43 (8) (2010) 2666-2680.

[10] J. Salvi, J. Pagès, J. Batlle, Pattern codification strategies in structured light systems, Pattern Recognit. 37 (4) (2004) 827-849.

[11] J. Pages, J. Salvi, R. Garcia, C. Matabosch, Overview of coded light projection techniques for automatic 3D profiling, IEEE Int. Conf. Robot. Autom. 1 (2003) 133-138. 
[12] D. Scharstein, R. Szeliski, High-accuracy stereo depth maps using structured light, Proc. IEEE Comput. Soc. Conf. Comput. Vis. Pattern Recognit. (2003) 195-202.

[13] V. Niola, C. Rossi, S. Savino, S. Strano, A method for the calibration of a 3-d laser scanner, Robot. Comput.-Integr. Manuf. 27 (2) (2011) 479-484.

[14] F. Zhou, G. Zhang, Complete calibration of a structured light stripe vision sensor through planar target of unknown orientations, Image Vis. Comput. 23 (1) (2005) 59-67.

[15] Z. Wei, F. Zhou, G. Zhang, 3d coordinates measurement based on structured light sensor, Sens. Actuators A, Phys. 120 (2) (2005) 527-535.

[16] R. Furukawa, H. Kawasaki, Laser range scanner based on self-calibration techniques using coplanarities and metric constraints, Comput. Vis. Image Underst. 113 (2009) 1118-1129.

[17] J. Park, G. DeSouza, A. Kak, Dual-beam structured-light scanning for 3-d object modeling, in: Proceedings of the 3rd International Conference on 3-D Digital Imaging and Modeling, IEEE Computer Society, 2001, pp. 65-72.

[18] S. Ozan, S. Gumustekin, Calibration of a 3d environment scanner with a pair of line lasers, in: 20th Conference on Signal Processing and Communications Applications, SIU2012 (in Turkish).

[19] E. Trucco, A. Verri, Introductory Techniques for 3-D Computer Vision, Prentice Hall PTR, Upper Saddle River, NJ, USA, 1998.

[20] J.Y. Bouguet, Camera calibration toolbox for Matlab, http://www.vision. caltech.edu/bouguetj.

[21] Z. Zhang, Flexible camera calibration by viewing a plane from unknown orientations, in: IEEE International Conference on Computer Vision, ICCV'99, 1999, pp. 666-673.

[22] E.W. Dijkstra, A note on two problems in connexion with graphs, Numer. Math. 1 (1959) 269-271

[23] M. Izquierdo, M. Sanchez, A. Ibañez, L. Ullate, Sub-pixel measurement of 3d surfaces by laser scanning, Sens. Actuators A, Phys. 76 (1999) 1-8.

[24] K. Okada, S. Kagami, M. Inaba, H. Inoue, Plane segment finder: algorithm, implementation and applications, IEEE Int. Conf. Robot. Autom. 2 (2001) 2120-2125.

[25] D. Borrmann, J. Elseberg, K. Lingemann, A. Nüchter, The 3d hough transform for plane detection in point clouds: A review and a new accumulator design, 3D Res. 2 (2011) 32:1-32:13.

[26] G. Strang, Computational Science and Engineering, Wellesley Cambridge Press, 2007.
[27] J.A. Nelder, R. Mead, A simplex method for function minimization, Comput. J. 7 (1965) 308-313.

[28] H. Zhao, A fast sweeping method for eikonal equations, Math. Comput. 74 (250) (2004) 603-627.

[29] H. Zhao, S. Osher, B. Merriman, M. Kang, Implicit, nonparametric shape reconstruction from unorganized points using a variational level set method, Comput. Vis. Image Underst. 80 (1998) 295-319.

[30] W. Lorensen, H. Cline, Marching cubes: A high resolution 3d surface construction algorithm, SIGGRAPH Comput. Graph. 21 (4) (1987) 163-169.

[31] E.K. Chong, S.H. Żak, An Introduction to Optimization, John Wiley \& Sons, Inc., 2001.

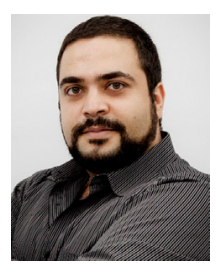

Sükrü Ozan received his M.S. degree in Electronics and Communication Engineering from Izmir Institute of Technology, Izmir/Turkey and B.S. degree in Electrical and Electronics Engineering from Middle East Technical University, Ankara/Turkey. Currently, he is a Ph.D. candidate in Izmir Institute of Technology. His current research is focused on vision based reconstruction of geometrical and physical properties of surfaces. He is also a co-founder of a Google Certified Partner Company named AdresGezgini which has been serving in Turkey since 2007.

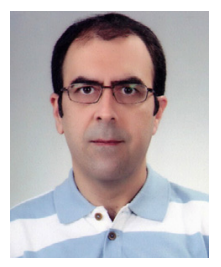

Şevket Gümüștekin received his Ph.D. and M.S. degrees in Electrical Engineering from University of Pittsburgh, PA/USA, B.S. degree in Electronics Engineering from Hacettepe University, Ankara/Turkey. He has been a faculty member in Izmir Institute of Technology, Department of Electrical and Electronics Engineering since 2000 . He has been involved in scientific and industrial projects in image analysis and display systems. His current research interests include augmented reality, computer vision systems and applications. 